\title{
Matrine alters microRNA expression profiles in SGC-7901 human gastric cancer cells
}

\author{
HAILONG LI ${ }^{1,2^{*}}$, SHOUPIN XIE ${ }^{3 *}$, XIAOJUN LIU ${ }^{4}$, HONGYAN WU ${ }^{1,2}$, \\ XINGYAO LIN ${ }^{1,2}$, JING GU ${ }^{1,2}$, HUPING WANG ${ }^{1,2}$ and YONGQIANG DUAN ${ }^{1}$ \\ ${ }^{1}$ Key Laboratory of TCM Pharmacology and Toxicology of Gansu Province, Gansu Traditional Chinese \\ Medical University; ${ }^{2}$ New Products of Traditional Chinese Medicine Engineering Laboratory of Gansu Province, \\ Lanzhou, Gansu 730000; ${ }^{3}$ First People's Hospital of Lanzhou City, Lanzhou, Gansu 730050; ${ }^{4}$ Oncology Department \\ of the First Hospital of Lanzhou University, Lanzhou, Gansu 730000, P.R. China
}

Received May 2, 2014; Accepted August 3, 2014

DOI: $10.3892 /$ or_xxxxxxxx

\begin{abstract}
Matrine, a major alkaloid extracted from Sophora flavescens, has been reported to possess antitumor properties in several types of cancers, including gastric cancer. However, its mechanisms of action on gastric cancer remain poorly understood. Dysregulation of microRNAs, a class of small, non-coding, regulatory RNA molecules involved in gene expression, is strongly correlated with cancer. The aim of the present study was to demonstrate that matrine treatment altered miRNA expression in SGC7901 cells. Using miRCURY ${ }^{\mathrm{TM}}$ microarray analysis, we identified 128 miRNAs substantially exhibiting $>2$-fold expression changes in matrine-treated cells relative to their expression levels in untreated cells. RT-qPCR was used to show that the levels of 8 miRNAs whose target genes were clustered in the cell cycle pathway increased, while levels of 14 miRNAs whose target genes were clustered in the MAPK signaling pathway decreased. These results were consistent with those from the miRNA microarray experiment. Bioinformatical analysis revealed that the majority of 57 identified enrichment pathways were highly involved in tumorigenesis. In conclusion, the results demonstrated that matrine induces considerable changes in the miRNA expression profiles of SGC7901 cells, suggesting miRNA microarray combined with RT-qPCR validation and bioinformatical analysis provide a novel and promising approach to identify anticancer targets and the mechanisms of matrine involved.
\end{abstract}

\section{Introduction}

Matrine is a major alkaloid extracted from Sophora flavescens that has been used clinically in the treatment of gastric

Correspondence to: Professor Yongqiang Duan, School of Basic Medical Sciences, Gansu Traditional Chinese Medical University, 35 Dingxi Road, Lanzhou, Gansu 730000, P.R. China

E-mail: gslz860931@163.com; dyqgs2008@163.com

${ }^{*}$ Contributed equally

Key words: miRNA, microarray profile, matrine, gastric cancer cancer. Matrine exhibits antitumor activity in several types of cancer including gastric (1-5), lung cancer $(6,7)$, acute myeloid leukemia $(8,9)$, breast $(10)$, prostate $(11)$, hepatocellular (12), pancreatic (13), colon cancer (14) and gallbladder carcinomas (15). The anticancer properties of matrine are associated with its ability to suppress proliferation and induce apoptosis in tumor cell lines through a variety of different pathways $(2,7-10,16)$. However, in the case of gastric cancer the mechanism of action has yet to be clarified.

Matrine injections have been found to inhibit the migration, invasion and adhesion capacity of SGC-7901 gastric cancer cells in vitro. This inhibition may be correlated with the downregulation of CD44 (V6) protein expression (2). Matrine injections are also able to induce apoptosis of SGC-7901 cells by upregulating Fas/FasL expression and activating caspase-3 (3). Furthermore, matrine can induce apoptosis in MKN45 gastric cancer cells via an increase in pro-apoptotic molecules from the Bcl-2 family (5). Matrine has been found to modulate $\mathrm{NF}-\kappa \mathrm{B}, \mathrm{XIAP}, \mathrm{CIAP}$ and $\mathrm{p}-\mathrm{ERK}$ protein expression in the MNK45 cell line, resulting in antitumor effects (4). In addition, matrine inhibits the adhesion and migration of BGC823 gastric cancer cells by affecting the structure and function of the vasodilator-stimulated phosphoprotein (VASP) (17).

It has recently been reported that matrine suppresses breast cancer by downregulating miR-21, leading to the dephosphorylation of Akt and resulting in an accumulation of Bad, p21(/WAF1/CIP1) and p27(/KIP1) (10). Thus, miRNA may act as a mediator, contributing to the therapeutic efficacy of matrine. The aim of the present study was to examine the effects of matrine on miRNA expression profiles of the SGC7901 gastric cancer cell line and to identify the putative target genes by analyzing differentially expressed miRNAs and the enrichment pathways of target genes. To the best of our knowledge, this is the first study to examine the effect of treatment with matrine on miRNA expression profiles in gastric cancer cells.

\section{Materials and methods}

Cell line and cell culture. The SGC7901 cell line was obtained from the Cancer Institute and Hospital, Chinese 
Academy of Medical Sciences (CAMS) (Beijing, China). The cells were grown in $90 \%$ RPMI-1640, supplemented with $10 \% \mathrm{FBS}, 100 \mathrm{U} / \mathrm{ml}$ penicillin, and $100 \mathrm{mg} / \mathrm{ml}$ streptomycin and incubated at $37^{\circ} \mathrm{C}$ in a humidified atmosphere of $5 \% \mathrm{CO}_{2}$.

Matrine treatment. Matrine (Baoji Fangsheng Pharmaceutical Co., Ltd., Xian, China) was analyzed by HPLC assay and dissolved in phosphate-buffered saline (PBS; Invitrogen-Gibco, Grand Island, NY, USA). SGC-7901 cell cultures were then treated for $24 \mathrm{~h}$ with $0.5,1.0,1.5,2.0$ and $2.5 \mathrm{mg} / \mathrm{ml}$ concentrations of matrine. Subsequently, $20 \mu 1$ of $5 \mathrm{mg} / \mathrm{ml}$ MTT in PBS was added to each well and the plate was incubated at $37^{\circ} \mathrm{C}$ for $4 \mathrm{~h}$. The plate was then centrifuged at $1,000 \mathrm{x} \mathrm{g}$ for $2 \mathrm{~min}$ and followed by removal of the medium. One-hundred and fifty microliter of dimethyl sulfoxide (DMSO; Sigma, St. Louis, MO, USA) was then added. After incubation at $37^{\circ} \mathrm{C}$ for 5 min, absorbance in the control and matrine-treated cells was measured spectrophotometrically at $570 \mathrm{~nm}$ using a benchmark microtiter plate reader (Bio-Rad Laboratories, Hercules, CA, USA). Microarray array was subsequently conducted on the control (blank) vs. $1.5 \mathrm{mg} / \mathrm{ml}$ matrine (MA) treatment groups.

miRNA microarray analysis. Total RNA, including miRNA, was isolated using the miRNeasy mini kit (Qiagen, Hilgen, Germany), according to the manufacturer's instructions. RNA quality and quantity was measured via a NanoDrop spectrophotometer (ND-1000; NanoDrop Technologies, Wilmington, DE, USA) and RNA integrity was determined by gel electrophoresis. The concentration and purity of total RNA were assessed at absorbance readings of 260 and $280 \mathrm{~nm}$, using an ultraviolet spectrophotometer. Only the RNA samples with ratios of A260/A280 >1.8 were used in the present study. After isolating the RNA, the miRCURY Hy $3^{\mathrm{TM}} / \mathrm{Hy} 5^{\mathrm{TM}}$ Power labeling kit (Exiqon, Copenhagen, Denmark) was used for miRNA labeling, according to the manufacturer's instructions. One microgram of each sample was 3'-end-labeled with a $\mathrm{Hy}^{\mathrm{TM}}$ fluorescent label using T4 RNA ligase, as follows: RNA was placed in $2.0 \mu \mathrm{l}$ of water and combined with $1.0 \mu \mathrm{l}$ of CIP buffer and CIP (Exiqon, Vedbaek, Denmark). After incubating for $30 \mathrm{~min}$ at $37^{\circ} \mathrm{C}$ the reaction was terminated by 5 -min incubation at $95^{\circ} \mathrm{C}$. A volume of $3.0 \mu \mathrm{l}$ labeling buffer, $1.5 \mu \mathrm{l}$ fluorescent label (Hy3 $3^{\mathrm{TM}}$ ), 2.0 $\mu \mathrm{l}$ DMSO, and $2.0 \mu \mathrm{l}$ labeling enzyme were then added. The mixture was incubated for $1 \mathrm{~h}$ at $16^{\circ} \mathrm{C}$ for labeling, followed by 15 -min incubation at $65^{\circ} \mathrm{C}$ to terminate the labeling reaction. After labeling, the Hy $3^{\mathrm{TM}}$-labeled samples were hybridized on the miRCURY ${ }^{\mathrm{TM}}$ LNA Array v.18.0 (Exiqon, Copenhagen, Denmark), according to the array manual. A $25 \mu \mathrm{l}$ volume of the $\mathrm{Hy} 3^{\mathrm{TM}}$-labeled sample mixture and $25 \mu \mathrm{l}$ hybridization buffer were denatured for $2 \mathrm{~min}$ at $95^{\circ} \mathrm{C}$, incubated on ice for 2 min and then hybridized to the microarray for $16-20 \mathrm{~h}$ at $56^{\circ} \mathrm{C}$ in a 12 -Bay hybridization system (NimbleGen Hybridization System 12; Roche Applied Science, Madison, WI, USA), which provides an active mixing action and constant incubation temperature to improve hybridization uniformity and signal enhancement. Following hybridization, slides were prepared, washed several times using a wash buffer kit (Exiqon), and dried via centrifugation for $5 \mathrm{~min}$ at $400 \mathrm{rpm}$. The slides were scanned using an Axon GenePix 4000B microarray scanner (Axon Instruments, Foster City, CA, USA).
miRNA target gene prediction and signaling pathway analyses. miRBase, miRanda and TargetScan were used to predict the target genes of differentially expressed miRNAs following matrine treatment. The prediction information from the three databases that overlapped was considered the final result. The miRNA target gene prediction databases miRFocus 2.0 (http://mirfocus.org/index.php) and miRWalk (http://www. umm.uni-heidelberg.de/apps/zmf/mirwalk/) were also used to analyze plausible KEGG pathway enrichment of dysregulated miRNA targets. The miRFocus 2.0 prediction tools included miRanda, MirTarget2, PicTar, microT and TargetScanS. The experimental validated target gene tools included miRecords, miR2Disease, TarBase and miRTarBase. The prediction databases support number was $\geq 3$ and the Fisher test P-value cut-off was 0.01 .

miRNA quantification by RT-qPCR. A SYBR-Green RT-PCR assay was used for miRNA quantification. In brief, $1 \mu \mathrm{g}$ of total RNA, containing miRNA, was polyadenylated using poly(A) polymerase and was reverse transcribed to cDNA using a miScript Reverse Transcription kit, according to the manufacturer's instructions (GeneCopoeia, Rockville, MD, USA). The miScript Universal primer was provided by the manufacturer (GeneCopoeia) and the miScript SYBR-Green PCR kit was used. RT-PCR was performed using the Bio-Rad CFX96 real-time PCR system. Each reaction was performed in a final volume of $10 \mu \mathrm{l}$ containing $2 \mu \mathrm{l} \mathrm{cDNA}, 0.5 \mathrm{mM}$ of each primer and 1X SYBR-Green PCR Master Mix (GeneCopoeia). The amplification program was as follows: denaturation at $95^{\circ} \mathrm{C}$ for $10 \mathrm{~min}$, followed by $45 \mathrm{cycles}$ at $94^{\circ} \mathrm{C}$ for $15 \mathrm{sec}, 55^{\circ} \mathrm{C}$ for $30 \mathrm{sec}$ and $70^{\circ} \mathrm{C}$ for $30 \mathrm{sec}$, in which fluorescence was obtained. After completion of the PCR cycles melting curve analyses and electrophoresis, on $2.5 \%$ agarose gels were performed to validate the specificity of the expected PCR product. Each sample was analyzed in triplicate. The expression levels of miRNAs were normalized to RNU6B and the relative gene expression was calculated as 2-(CTMiRNA-CTRNU6BRNA) . Each sample was analyzed in triplicate. All the miRNA PCR primers were purchased from GeneCopoeia.

Statistical analysis. Scanned images of the miRNA Array were imported into GenePix Pro 6.0 software (Axon) for grid alignment and data extraction. Replicated miRNAs were averaged and miRNAs with intensities of $\geq 50$ in all the samples were used to calculate the normalization factor. Expressed data were normalized using the Median normalization. After normalization, differentially expressed miRNAs were identified through fold change filtering. Hierarchical clustering was performed using MeV software (v4.9, TIGR). Results of realtime RT-PCR experiments are expressed as means \pm SD.

\section{Results}

MTT results. The antitumor reducing activity of matrine cytotoxicity $(0.5,1.0,1.5,2.0$ and $2.5 \mathrm{mg} / \mathrm{ml})$ to SGC-7901 cells culture is shown in Fig. 1. Matrine showed a dose-dependent inhibition of the growth of SGC-7901 cells.

Effects of matrine on miRNA expression profiling of SGC-7901 gastric cancer cells. After SGC-7901 cells were treated with 
Table I. Expression of 8 upregulated miRNAs altered by microarray experiment and RT-qPCR experiment validation after matrine treatment $(1.50 \mathrm{mg} / \mathrm{ml})$.

\begin{tabular}{lcc}
\hline & $\begin{array}{c}\text { Fold change, } \\
\text { microarray } \\
\text { experiment }\end{array}$ & $\begin{array}{c}\text { Fold change compared } \\
\text { with control; } \\
\text { mean } \pm \text { SD, } \\
\text { RT-qPCR experiment }\end{array}$ \\
\hline miR-192-5p & 6.352408 & $5.30 \pm 0.70$ \\
miR-194-5p & 3.258979 & $3.71 \pm 0.29$ \\
miR-299-5p & 4.094039 & $5.22 \pm 0.37$ \\
miR-300 & 4.702027 & $5.53 \pm 0.59$ \\
miR-302a-3p & 2.215804 & $4.28 \pm 0.81$ \\
miR-483-5p & 2.921674 & $3.60 \pm 0.82$ \\
miR-498 & 3.790496 & $5.34 \pm 0.40$ \\
miR-513a-5p & 2.628494 & $3.24 \pm 0.96$
\end{tabular}

(Fold change, mean $\pm \mathrm{SD}$, treated with matrine vs. untreated). Eight upregulated miRNAs found by miRNA microarray experiment were consistent with qPCR experiment validation. Relative gene expression was calculated as 2-(CTmiRNA-CTRNU6B RNA), and each sample was analyzed in triplicate.

Table II. Expression of 14 downregulated miRNAs altered by microarray experiment and qRT-PCR experiment validation after matrine treatment $(1.50 \mathrm{mg} / \mathrm{ml})$.

\begin{tabular}{lcc}
\hline miRNA & $\begin{array}{c}\text { Fold change, } \\
\text { microarray } \\
\text { experiment }\end{array}$ & $\begin{array}{c}\text { Fold change compared } \\
\text { with control; } \\
\text { mean } \pm \text { SD, } \\
\text { qRT-PCR experiment }\end{array}$ \\
\hline let-7b-5p & 0.458117 & $0.74 \pm 0.10$ \\
let-7c & 0.376421 & $0.67 \pm 0.07$ \\
let-7d-5p & 0.445587 & $0.09 \pm 0.01$ \\
let-7f-5p & 0.145997 & $0.76 \pm 0.13$ \\
miR-19a-3p & 0.327783 & $0.56 \pm 0.07$ \\
miR-19b-3p & 0.498536 & $0.60 \pm 0.07$ \\
miR-20b-5p & 0.218928 & $0.66 \pm 0.12$ \\
miR-21-5p & 0.341840 & $0.58 \pm 0.08$ \\
miR-27a-3p & 0.300471 & $0.56 \pm 0.04$ \\
miR-27b-3p & 0.432703 & $0.51 \pm 0.05$ \\
miR-29c-3p & 0.466145 & $0.78 \pm 0.07$ \\
miR-30e-5p & 0.489503 & $0.82 \pm 0.05$ \\
miR-98-5p & 0.386457 & $0.69 \pm 0.07$ \\
miR-106b-5p & 0.475087 & $0.53 \pm 0.08$ \\
\hline
\end{tabular}

(Fold change, treated with matrine vs. untreated). Results indicating the trend of 14 downregulated miRNAs found by miRNA microarray experiment was consistent with $\mathrm{qPCR}$ experiment validation. Relative gene expression was calculated as $2^{\text {-(CTmiRNA-CTRNU6B RNA) }}$, and each sample was analyzed in triplicate.

$1.5 \mathrm{mg} / \mathrm{ml}$ matrine for $24 \mathrm{~h}, 60 \mathrm{miRNAs}$ were downregulated (fold change, $\leq 0.5$ ) and 68 miRNAs were upregulated (fold change, $\geq 2.0$ ) (Fig. 2). By searching the miRFocus 2.0 and
Table III. Matrine-regulated miRNAs and the related documentation of expression in gastric cancer tissue or gastric cancer cell lines identified by microarray analysis or RT-qPCR experiments.

\begin{tabular}{|c|c|c|c|}
\hline $\begin{array}{l}\text { miRNA } \\
\text { name }\end{array}$ & $\begin{array}{l}\text { Matrine } \\
\text { treatment } \\
\text { response }\end{array}$ & $\begin{array}{l}\text { Expression } \\
\text { in gastric } \\
\text { cancer tissue } \\
\text { or cell lines }\end{array}$ & (Refs.) \\
\hline let-7b-5p & $\downarrow$ & $\uparrow$ & (18) \\
\hline miR-10a-5p & $\downarrow$ & $\uparrow$ & $(19,20)$ \\
\hline $\operatorname{miR}-15 b-5 p$ & $\downarrow$ & $\uparrow$ & (18) \\
\hline miR-18a-5p & $\downarrow$ & $\uparrow$ & $(21,22)$ \\
\hline miR-18b-5p & $\downarrow$ & $\uparrow$ & (21) \\
\hline $\operatorname{miR}-19 a-3 p$ & $\downarrow$ & $\uparrow$ & $(22,23)$ \\
\hline miR-19b-3p & $\downarrow$ & $\uparrow$ & $(18,20,24)$ \\
\hline miR-20b-5p & $\downarrow$ & $\uparrow$ & $(20,21,24,25)$ \\
\hline miR-21-5p & $\downarrow$ & $\uparrow$ & $(20,21,24,26-30)$ \\
\hline miR-23a-3p & $\downarrow$ & $\uparrow$ & $(20,26)$ \\
\hline miR-26b-5p & $\downarrow$ & $\uparrow$ & $(5,31)$ \\
\hline $\operatorname{miR}-27 a-3 p$ & $\downarrow$ & $\uparrow$ & $(20,25,26,32,33)$ \\
\hline miR-29b-3p & $\downarrow$ & $\downarrow$ & $(18,24)$ \\
\hline $\operatorname{miR}-29 c-3 p$ & $\uparrow$ & $\downarrow$ & $(18,24,34,35)$ \\
\hline miR-30e-5p & $\downarrow$ & $\downarrow$ & (20) \\
\hline miR-32-5p & $\downarrow$ & $\uparrow$ & (26) \\
\hline miR-34a-5p & $\downarrow$ & $\uparrow$ & $(20,22,36)$ \\
\hline $\operatorname{miR}-98$ & $\downarrow$ & $\uparrow$ & (22) \\
\hline miR-101-3p & $\downarrow$ & $\uparrow$ & (37) \\
\hline miR-106b-5p & $\downarrow$ & $\uparrow$ & $(20,22,30,36)$ \\
\hline $\operatorname{miR}-130 a-3 p$ & $\downarrow$ & $\downarrow$ & (30) \\
\hline $\operatorname{miR}-130 b-3 p$ & $\downarrow$ & $\downarrow$ & $(22,30)$ \\
\hline $\operatorname{miR}-148 b-3 p$ & $\downarrow$ & $\downarrow$ & $(24,37)$ \\
\hline $\operatorname{miR}-181 a-5 p$ & $\downarrow$ & $\uparrow$ & $(22,38,39)$ \\
\hline $\operatorname{miR}-338-3 p$ & $\downarrow$ & $\uparrow$ & $(25)$ \\
\hline miR-340-5p & $\uparrow$ & $\uparrow$ & $(21,22)$ \\
\hline miR-424-5p & $\uparrow$ & $\uparrow$ & (40) \\
\hline let-7a-5p & $\downarrow$ & $\uparrow$ & $(26,32,41-43)$ \\
\hline miR-192-5p & $\uparrow$ & $\uparrow$ & $(20,38,44)$ \\
\hline miR-194-5p & $\uparrow$ & $\uparrow$ & $(20,45)$ \\
\hline miR-335-5p & $\uparrow$ & $\uparrow$ & $(37,45)$ \\
\hline
\end{tabular}

Of 38 miRNAs that were downregulated after matrine treatment, 20 have been reported to be upregulated in human gastric cancers and gastric cancer cell lines and act as oncogenes. Additionally, 5 matrine-upregulated miRNAs were found to be elevated in human gastric cancers and gastric cancer cell lines, whereas 6 matrine-downregulated miRNAs were found to be reduced in human gastric cancers or cell lines.

miRWalk miRNA databases, only 38 of the 60 downregulated miRNAs and 8 of 68 upregulated miRNAs were annotated with their respective expressions in cancer tissues or cells or the predicted and validated target genes. Of the 46 annotated miRNAs, 25 were upregulated and 6 were downregulated in gastric cancer tissue or gastric cancer cell lines by miRNA microarray or RT-PCR (Tables I-III). 


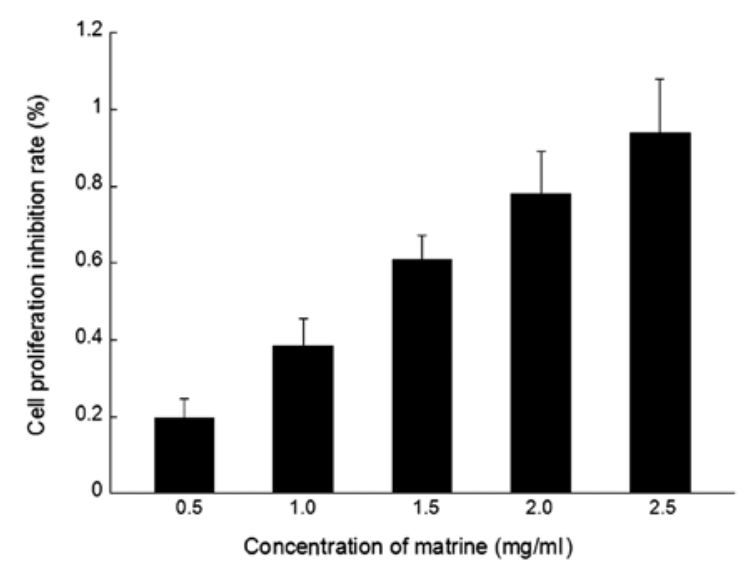

Figure 1. Matrine inhibited SGC-7901 cell proliferation in a dose-dependent manner. Cells were treated with $0.5,1.0,1.5,2.0$ and $2.5 \mathrm{mg} / \mathrm{ml}$ of matrine for $24 \mathrm{~h}$. Cell proliferation was determined by an MTT assay as described in 'Materials and methods'. The inhibition rate is expressed as the percentage of the cell inhibition rate compared with the control. Matrine inhibits SGC7901 cell proliferation. A statistically significant difference within different dosage groups is evident $(\mathrm{P}<0.01)$.
Signaling pathway analyses of differentially expressed miRNA target genes. The miRBase, miRanda and TargetScan bioinformatical databases were used to predict the target genes of 46 aberrantly expressed miRNAs. The results showed that there are 4,159 overlapped target genes as predicted by the three tools for all the downregulated miRNAs and 367 overlapped target genes for the upregulated miRNAs that exhibited a $>2$-fold expression change after matrine treatment (Fig. 3). Possible regulation mechanisms of differentially expressed miRNAs following matrine treatment were investigated using the bioinformatics database, miRFocus 2.0 , to select clustered enrichment KEGG pathways of plausible targets for each miRNA. A total of 57 enrichment pathways for predicted target genes were identified, including 36 enrichment signaling pathways for downregulated miRNAs. According to the Enrichment Score, the top 10 signaling pathways were the MAPK, focal adhesion, neurotrophin, axon guidance, ECM-receptor interaction, Wnt, amino sugar and nucleotide sugar metabolism, glycosaminoglycan biosynthesis-keratan
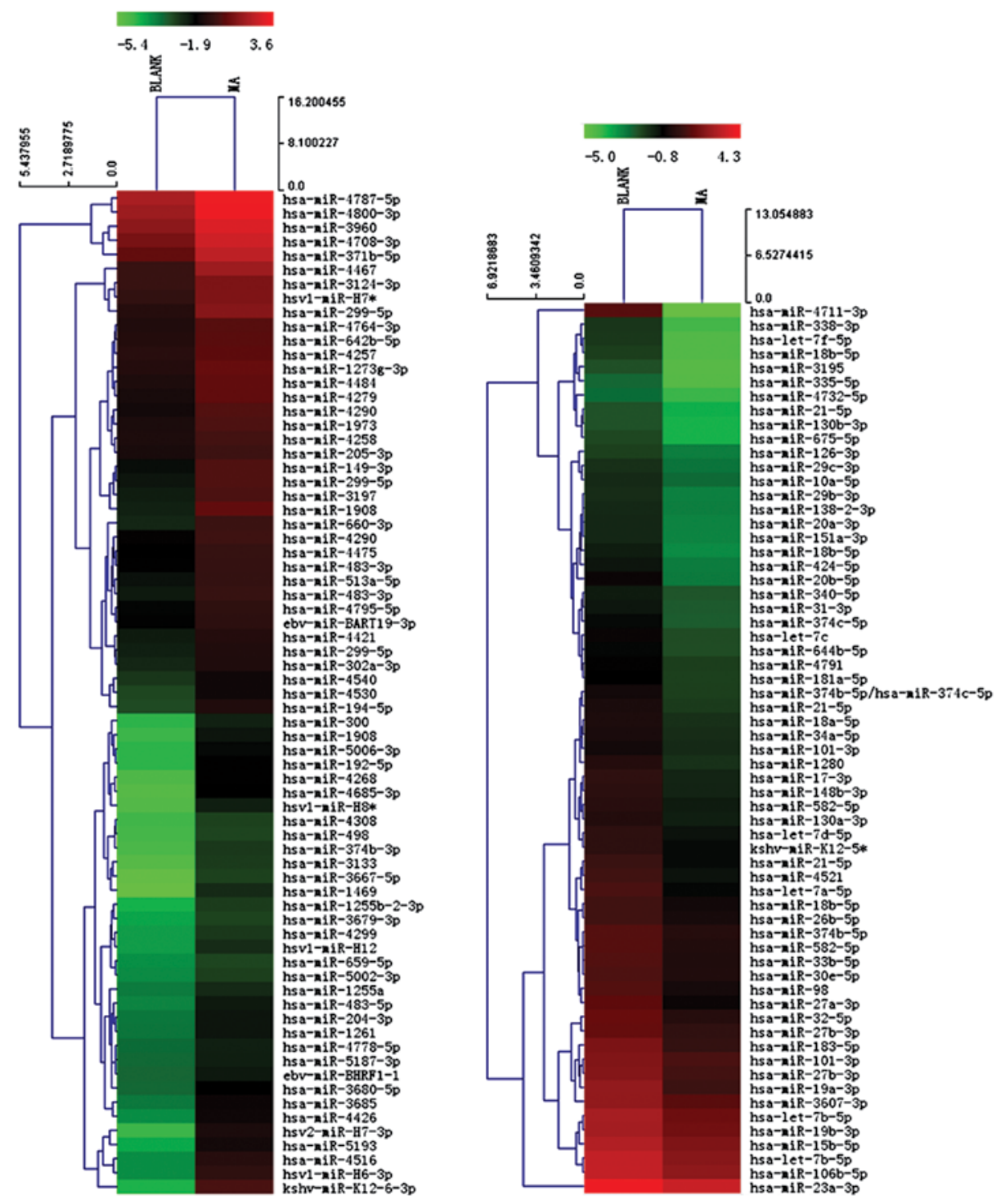

Figure 2. The miRNA expression profiles of SGC-7901 human gastric cancer cells after matrine treatment. A total of 128 human miRNAs including 60 upregulated and 68 downregulated exhibited $\geq 2$-fold change in expression, relative to the control cells. Relative miRNA expression is represented on a scale from -5.4 to 3.6, and -5.0 to 4.3 of up- and downregulated miRNAs, respectively, for each treatment group [control (blank), $1.5 \mathrm{mg} / \mathrm{ml} \mathrm{matrine} \mathrm{(MA)].}$ 
Table IV. Enrichment KEGG pathway analysis of downregulated miRNA target genes after matrine treatment.

\begin{tabular}{|c|c|c|c|c|}
\hline ID & Pathway definition & Fisher-P-value & FDR & Enrichment score \\
\hline hsa04010 & MAPK signaling pathway, Homo sapiens & 8.71694E-07 & 0.000218795 & 6.059636 \\
\hline hsa04510 & Focal adhesion, Homo sapiens & $6.70214 \mathrm{E}-06$ & 0.000841118 & 5.173787 \\
\hline hsa04722 & Neurotrophin signaling pathway, Homo sapiens & 4.59225E-05 & 0.00366527 & 4.337974 \\
\hline hsa04360 & Axon guidance, Homo sapiens & $6.48307 \mathrm{E}-05$ & 0.00366527 & 4.18822 \\
\hline hsa05200 & Pathways in cancer, Homo sapiens & 7.30133E-05 & 0.00366527 & 4.136598 \\
\hline hsa04512 & ECM-receptor interaction, Homo sapiens & 0.000645354 & 0.02684024 & 3.190202 \\
\hline hsa04310 & Wnt signaling pathway, Homo sapiens & 0.000748533 & 0.02684024 & 3.125789 \\
\hline hsa00520 & Amino sugar and nucleotide sugar metabolism & 0.001141903 & 0.03582719 & 2.942371 \\
\hline hsa00533 & Glycosaminoglycan biosynthesis, keratan sulfate & 0.001916047 & 0.05343643 & 2.717594 \\
\hline hsa04115 & p53 signaling pathway, Homo sapiens & 0.003151918 & 0.07764395 & 2.501425 \\
\hline hsa04930 & Type II diabetes mellitus, Homo sapiens & 0.003402723 & 0.07764395 & 2.468173 \\
\hline hsa04920 & Adipocytokine signaling pathway, Homo sapiens & 0.003740387 & 0.07823643 & 2.427083 \\
\hline hsa05211 & Renal cell carcinoma, Homo sapiens & 0.004416889 & 0.08527994 & 2.354883 \\
\hline hsa04141 & Protein processing in endoplasmic reticulum & 0.007499238 & 0.1175604 & 2.124983 \\
\hline hsa04330 & Notch signaling pathway, Homo sapiens & 0.007665283 & 0.1175604 & 2.115472 \\
\hline hsa04120 & Ubiquitin mediated proteolysis, Homo sapiens & 0.008175015 & 0.1175604 & 2.087511 \\
\hline hsa04070 & Phosphatidylinositol signaling system & 0.008771364 & 0.1175604 & 2.056933 \\
\hline hsa05014 & Amyotrophic lateral sclerosis (ALS) & 0.008897519 & 0.1175604 & 2.050731 \\
\hline hsa05202 & Transcriptional misregulation in cancer & 0.008898995 & 0.1175604 & 2.050659 \\
\hline hsa04974 & Protein digestion and absorption & 0.0100545 & 0.126184 & 1.99764 \\
\hline hsa04114 & Oocyte meiosis, Homo sapiens & 0.01317126 & 0.1534031 & 1.880373 \\
\hline hsa04728 & Dopaminergic synapse, Homo sapiens & 0.01344569 & 0.1534031 & 1.871417 \\
\hline hsa05164 & Influenza A, Homo sapiens & 0.01526479 & 0.1599239 & 1.816309 \\
\hline hsa03015 & mRNA surveillance pathway, Homo sapiens & 0.01529153 & 0.1599239 & 1.815549 \\
\hline hsa00770 & Pantothenate and CoA biosynthesis, Homo sapiens & 0.0196612 & 0.1973984 & 1.70639 \\
\hline hsa05219 & Bladder cancer, Homo sapiens & 0.02059202 & 0.1987922 & 1.686301 \\
\hline hsa05410 & Hypertrophic cardiomyopathy (HCM) & 0.02616797 & 0.2405538 & 1.58223 \\
\hline hsa04912 & GnRH signaling pathway, Homo sapiens & 0.02683468 & 0.2405538 & 1.571304 \\
\hline hsa04350 & TGF- $\beta$ signaling pathway, Homo sapiens & 0.02926174 & 0.2532654 & 1.5337 \\
\hline hsa05216 & Thyroid cancer, Homo sapiens & 0.03390439 & 0.2836667 & 1.469744 \\
\hline hsa04020 & Calcium signaling pathway, Homo sapiens & 0.03627392 & 0.2937018 & 1.440405 \\
\hline hsa04664 & Fc $\varepsilon$ RI signaling pathway, Homo sapiens & 0.04012037 & 0.3046442 & 1.396635 \\
\hline hsa04012 & ErbB signaling pathway, Homo sapiens & 0.04018206 & 0.3046442 & 1.395968 \\
\hline hsa04110 & Cell cycle, Homo sapiens & 0.04126655 & 0.3046442 & 1.384402 \\
\hline hsa04720 & Long-term potentiation, Homo sapiens & 0.04408158 & 0.3161279 & 1.355743 \\
\hline hsa00051 & Fructose and mannose metabolism, Homo sapiens & 0.04561514 & 0.3180389 & 1.340891 \\
\hline
\end{tabular}

'Fisher-P-value' stands for the enrichment P-value of the Pathway ID using the Fisher's exact test. 'FDR' stands for the false discovery rate of the Pathway ID. 'Enrichment score' stands for the Enrichment score value of the Pathway ID equaling '-log10 (P-value)'.

sulfate, and the p53 signaling pathway, as well as pathways in cancer (Table IV).

The remaining 21 enrichment signaling pathways were associated with the target genes of upregulated miRNAs. According to the Enrichment Score, the top 10 signaling pathways were the Legionellosis, notch, neurotrophin, protein processing in endoplasmic reticulum, TGF- $\beta$, small cell lung cancer, epithelial cell signaling in Helicobacter pylori infection, cell cycle, RIG-I-like and pertussis signaling pathways (Table V).

The miRFocus 2.0 database was thoroughly examined for target genes of pathways for MAPK signaling and cell cycles. The results showed that 196 genes are associated with MAPK signaling pathways regulated by the 14 downregulated miRNAs, while 53 genes are associated with cell cycle pathways regulated by the 8 upregulated miRNAs. In this study, of the 159 genes 14 miRNAs were downregulated (Figs. 4 and 5).

Quantification of dysregulated miRNAs whose target genes are clustered in MAPK signaling pathway and cell cycle by $R T-q P C R$. After SGC-7901 cells were treated with $1.5 \mathrm{mg} / \mathrm{ml}$ matrine for $24 \mathrm{~h}, 14$ miRNAs whose target genes were clustered in the MAPK signaling pathway and 8 miRNAs whose 
A

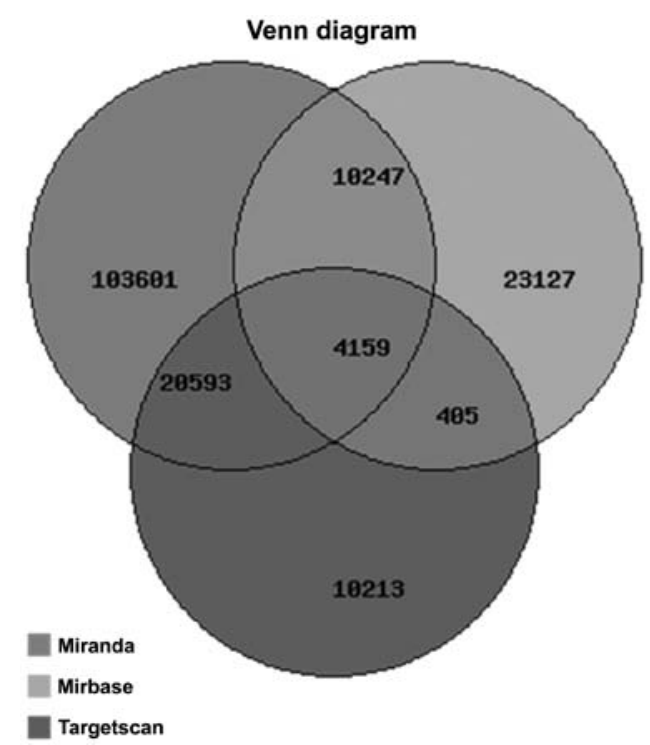

B

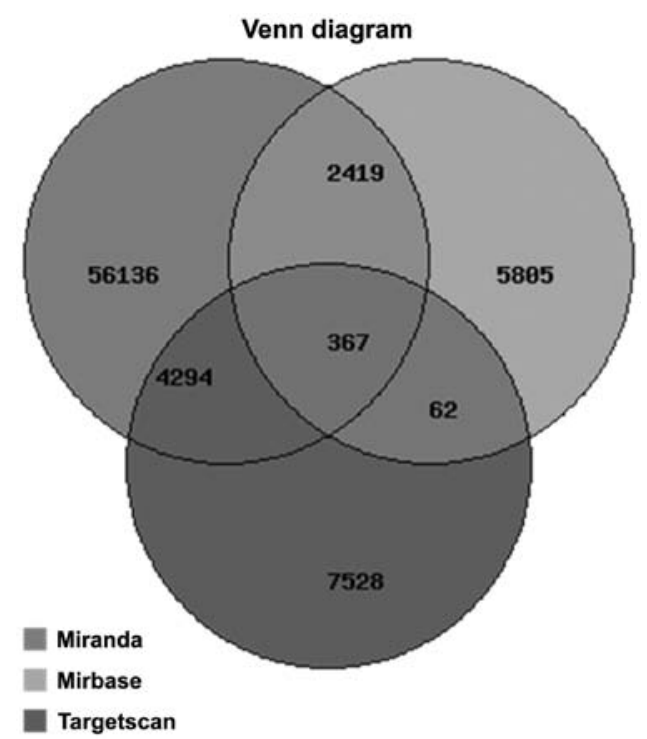

Figure 3. The Venn diagram shows the predicted miRNA target genes using the miRBase, miRanda and TargetScan bioinformatical databases. There are (A) 4,159 overlapped target genes as predicted by the three tools for all the downregulated miRNAs and (B) 367 overlapped target genes for the upregulated miRNAs that exhibited a $>2$-fold expression change after matrine treatment.

Table V. Enrichment KEGG pathway analysis of upregulated miRNA target genes after matrine treatment.

\begin{tabular}{lllll}
\hline ID & \multicolumn{1}{c}{ Pathway definition } & Fisher-P-value & FDR & Enrichment score \\
\hline hsa05134 & Legionellosis, Homo sapiens & 0.000904176 & 0.2269482 & 3.043747 \\
hsa04330 & Notch signaling pathway, Homo sapiens & 0.002738301 & 0.3436568 & 2.562519 \\
hsa04722 & Neurotrophin signaling pathway, Homo sapiens & 0.004751378 & 0.3842182 & 2.32318 \\
hsa04141 & Protein processing in endoplasmic reticulum & 0.007446489 & 0.3842182 & 2.128048 \\
hsa04350 & TGF- $\beta$ signaling pathway, Homo sapiens & 0.00777451 & 0.3842182 & 2.109327 \\
hsa05222 & Small cell lung cancer, Homo sapiens & 0.009184498 & 0.3842182 & 2.036945 \\
hsa05120 & Epithelial cell signaling in Helicobacter pylori infection & 0.01317173 & 0.4225359 & 1.880357 \\
hsa04110 & Cell cycle, Homo sapiens & 0.01437375 & 0.4225359 & 1.84243 \\
hsa04622 & RIG-I-like receptor signaling pathway & 0.01566376 & 0.4225359 & 1.805104 \\
hsa05133 & Pertussis, Homo sapiens & 0.01845303 & 0.4225359 & 1.733932 \\
hsa04620 & Toll-like receptor signaling pathway & 0.01905906 & 0.4225359 & 1.719898 \\
hsa05166 & HTLV-I infection, Homo sapiens & 0.02046761 & 0.4225359 & 1.688933 \\
hsa04010 & MAPK signaling pathway, Homo sapiens & 0.0231678 & 0.4225359 & 1.635115 \\
hsa04120 & Ubiquitin mediated proteolysis, Homo sapiens & 0.02356774 & 0.4225359 & 1.627682 \\
hsa05110 & Vibrio cholerae infection, Homo sapiens & 0.02532895 & 0.4238377 & 1.596383 \\
hsa04512 & ECM-receptor interaction, Homo sapiens & 0.03141567 & 0.4711115 & 1.502854 \\
hsa04621 & NOD-like receptor signaling pathway, Homo sapiens & 0.03190795 & 0.4711115 & 1.496101 \\
hsa04310 & Wnt signaling pathway, Homo sapiens & 0.03732464 & 0.494149 & 1.428004 \\
hsa05131 & Shigellosis, Homo sapiens & 0.03744232 & 0.494149 & 1.426637 \\
hsa05200 & Pathways in cancer, Homo sapiens & 0.03937442 & 0.494149 & 1.404786 \\
hsa04721 & Synaptic vesicle cycle,Homo sapiens & 0.04349567 & 0.5198769 & 1.361554 \\
\hline
\end{tabular}

'Fisher-P-value' stands for the enrichment P-value of the Pathway ID using the Fisher's exact test. 'FDR' stands for the false discovery rate of the Pathway ID. 'Enrichment score' stands for the Enrichment score value of the Pathway ID equaling '-log10 (P-value)'.

target genes are clustered in pathways of the cell cycle were selected to verify their expression using RT-qPCR. The results showed the alterations of 8 upregulated miRNAs, including miR-192-5p, miR-299-5p, miR-194-5p, miR-300, miR-302a-3p,
miR-483-5p, miR-498 and miR-513a-5p whose target genes were clustered in the cell cycle pathway following matrine treatment $(1.5 \mathrm{mg} / \mathrm{ml})$, were consistent with alterations identified by miRNA microarray. However, for the 38 down-regulated 


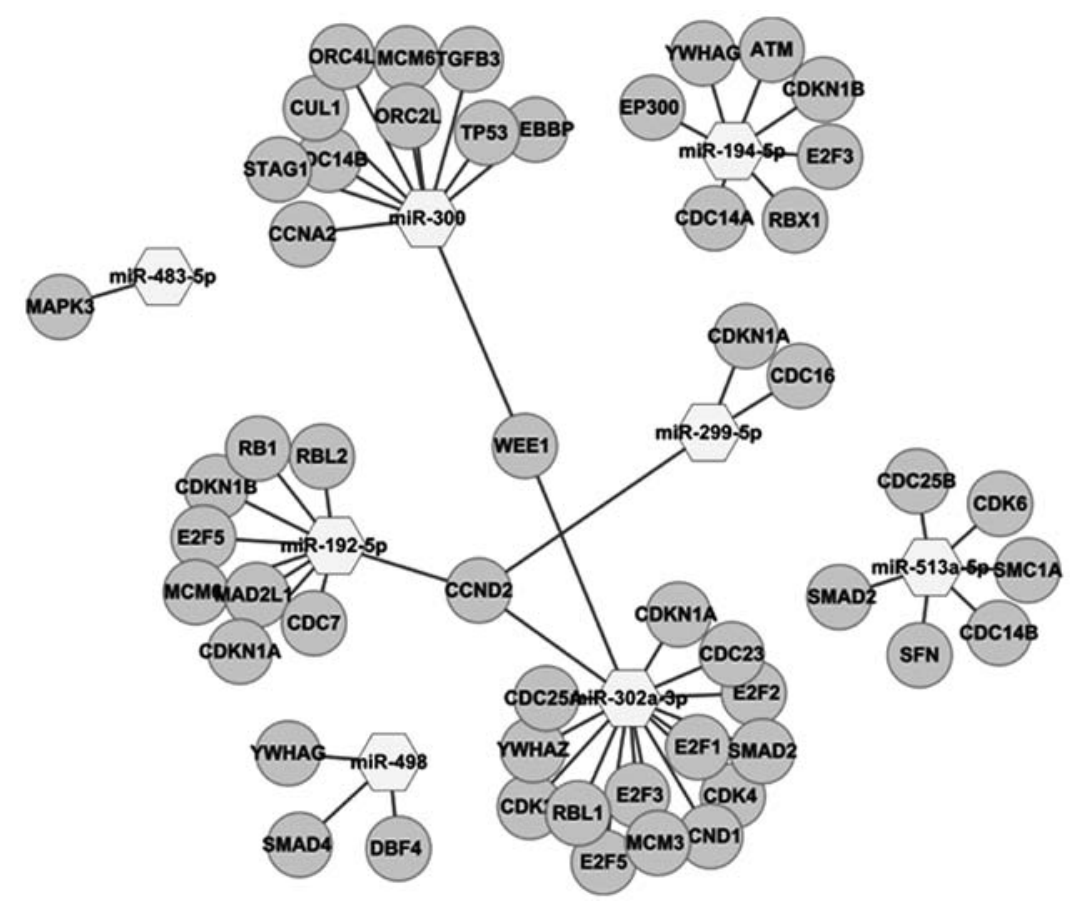

Figure 4. Predicted and validated cell cycle pathway (hsa04110) targets of 8 upregulated miRNAs that exhibited a $>2$-fold expression change in response to matrine, following a search in the miRfocus 2.0 and miRWalk databases. This graph was generated by Cytoscape 2.8. The hexagon node represents miRNAs, and the elliptical node represents target genes.

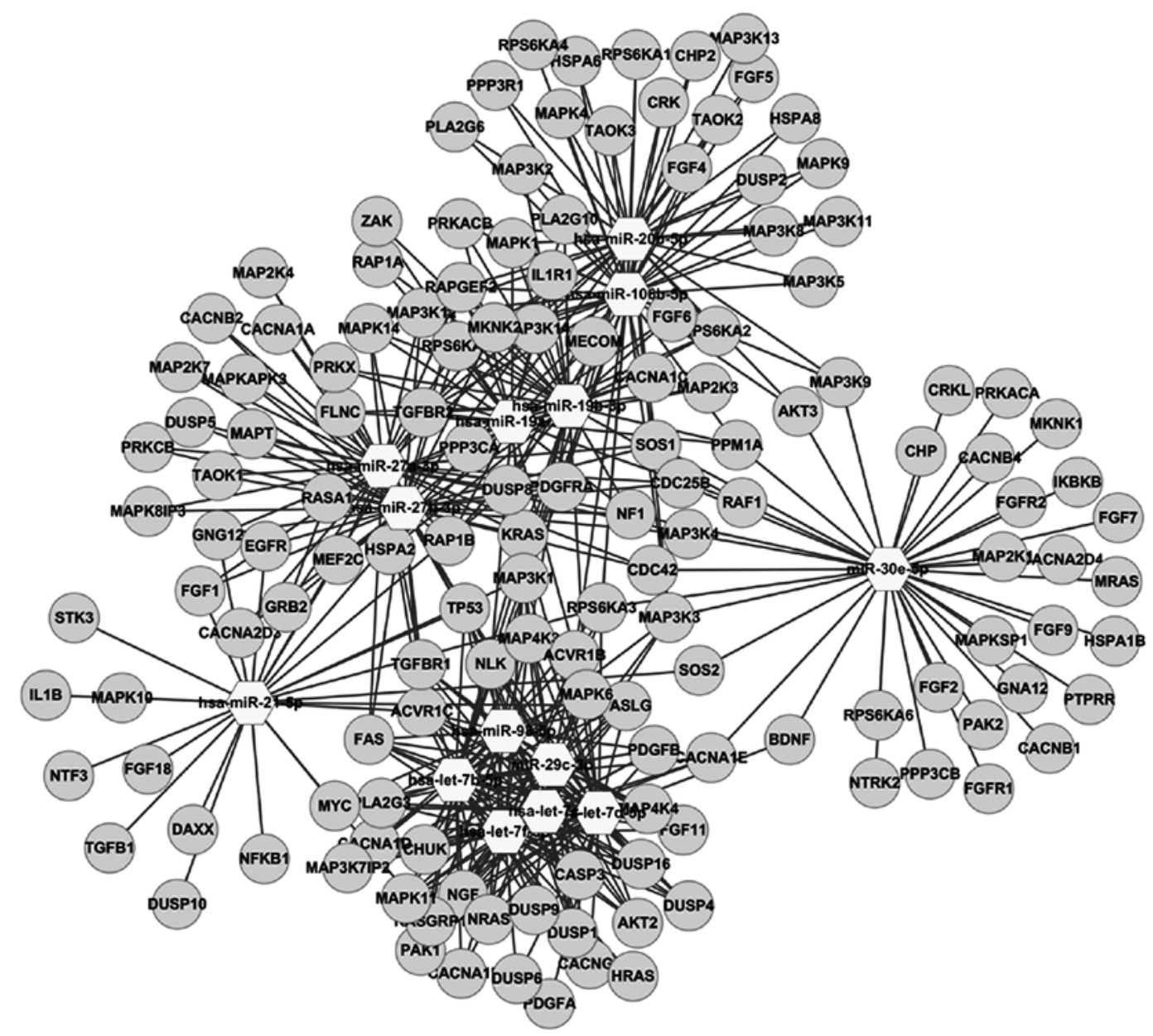

Figure 5. Predicted and validated MAPK signaling (hsa04010) pathway targets of 14 downregulated miRNAs that exhibited a $>2$-fold expression change in response to matrine, following a search in the miRfocus 2.0 and miRWalk databases. This graph was generated by Cytoscape 2.8 . The hexagon node represents miRNAs, and the elliptical node represents target genes. 
miRNAs, the alterations of 14 miRNAs following matrine treatment $(1.5 \mathrm{mg} / \mathrm{ml})$, including let-7b-5p, let-7c, let-7d-5p, let-7f-5p, miR-19a-3p, miR-19b-3p, miR-20b-5p, miR-21-5p, miR-27a-3p, miR-27b-3p, miR-29c-3p, miR-30e-5p, miR-98-5p and $\mathrm{miR}-106 \mathrm{~b}-5 \mathrm{p}$ whose target genes were clustered in the MAPK signaling pathway, were consistent with alterations identified by miRNA microarray (Tables I and II).

\section{Discussion}

Based on the miRNA microarray analysis, 128 miRNAs with differential expression were identified after $24 \mathrm{~h}$ of treatment with $1.5 \mathrm{mg} / \mathrm{ml}$ matrine. Of these, 68 were upregulated and 60 were downregulated following matrine treatment. The functions of 8 of the upregulated miRNAs and 38 of the downregulated miRNAs have been annotated with their target genes in the miRbase miRNA database. Of these 46 differentially expressed miRNAs with annotations, results of RT-qPCR showed that 8 miRNAs whose target genes were clustered in the cell cycle pathway were upregulated after matrine treatment, while 14 miRNAs whose target genes were clustered in the MAPK signaling pathway were downregulated after matrine treatment.

The majority of the miRNAs that were affected by matrine treatment were associated with the pathogenesis and development of human gastric cancers. Of the 38 miRNAs that were downregulated after matrine treatment, 20 were upregulated in human gastric cancers and gastric cancer cell lines and act as oncogenes. These include let-7b-5p, miR-10a-5p, miR-15b-5p, miR-18a-5p, miR-18b-5p, miR-19a-3p, miR-19b-3p, miR20b-5p, miR-21-5p, miR-23a-3p, miR-26b-5p, miR-27a-3p, miR-27b-3p, miR-32-5p, miR-34a-5p, miR-98, miR-106b-5p, miR-181a-5p, miR-183-5p and miR-338-3p. Notably, matrine upregulated 5 miRNAs that have been reported to be elevated in human gastric cancers and gastric cancer cell lines. These include let-7a-5p, miR-192-5p, miR-194-5p, miR-335-5p and miR-424-5p. In addition, matrine downregulated 6 miRNAs that have been reported to be reduced in human gastric cancers and gastric cancer cell lines. These include miR-29b-3p, miR-29c-3p, miR-30e-5p, miR-130a-3p, 130b-3p and 148b-3p (Table III) (7-34). Bioinformatical analysis of differentially expressed miRNAs revealed that the target genes were associated with cancer and cell cycle pathways, as well as the MAPK, p53, TGF- $\beta$, mTOR, Wnt and notch signaling pathways, which are involved in tumorigenesis. The 8 upregulated miRNAs and 14 downregulated miRNAs, in particular, are involved in the cell cycle and MAPK signaling pathways and were found to be regulated by matrine. Thus, gastric, as well as other cancer cells may be mediated by miRNAs.

Since matrine promotes the apoptosis of Raji cells by activated p38MAPK (46), it suppressed the activity of ERK and increased the activities of p38 and JNK when inhibiting the proliferation of the U937 cell line, inducing apoptosis in vitro (47). Furthermore, matrine inhibited the migration of HUVECs induced by A549 by suppressing MAPK/ERK signaling transduction (47). In addition, matrine has been reported to reduce the percentage of cells in the G2/M phase, and increase the percentage of cells arrested in the G0/G1 phase of the cell cycle, resulting in blocking of the cell cycle at the G0/G1 stage when treating cancer cells, such as human
SGC-7901 gastric cancer cell line (1), HT29 colon cancer cell line (14), human rhabdomyosarcoma cells (48), PC-3 prostate cancer cell line (11), HepG2 human hepatoma cell line (12), human A549 adenocarcinoma lung cancer cell line (49) and the human K562 leukemia cell line (50). Therefore, the aberrantly expressed miRNAs whose target genes were clustered in the MAPK signaling pathway and pathways selected for analysis of their predicted and validated target genes by miRFocus 2.0 and miRWalk provide further explanations of the underlying mechanism of action and mechanisms involved in the SGC-7901 gastric cancer cell line following matrine treatment.

Putative target miRNAs, along with target genes and enriched KEGG pathways identified through analysis and validation of the differentially expressed miRNAs in human SGC-7901 gastric cancer cells that had been treated with matrine has facilitated the possibility of finding the target of natural, chemopreventive agents. This may provide insight into the antitumor mechanism of action of matrine, particularly in the case of gastric cancer. In recent years, there have been several reports of miRNA regulation via natural, non-toxic, chemopreventive agents, including curcumin, resveratrol, isoflavones, (-)-epigallocatechin-3-gallate (EGCG), lycopene, 3,3'-diindolylmethane (DIM) and indole-3-carbinol (I3C) $(51,52)$. Thus, natural agents, including matrine, may inhibit cancer progression, increase drug sensitivity, reverse epithelial-mesenchymal transition (EMT), and prevent metastasis though the modulation of miRNAs, providing promising new therapies for the treatment of cancer.

In conclusion, matrine affects miRNA expression in the SGC-7901 gastric cancer cell line as evidenced by miRNA microarray and analysis of differentially expressed miRNA target genes and enrichment pathways. These results, combined with RT-qPCR validation and bioinformatical analyses, provide a novel and promising approach to identify targets for matrine and/or other natural substances, when treating cancer.

\section{Acknowledgements}

We would like to thank KangChen Bio-teck (Shanghai, China) for microarray experiments. The present study was supported by the Key Laboratory of TCM Pharmacology and Toxicology of Gansu Province under the Grant Open Plan (no: ZDSYS-KJ-2012-003, ZDSYS-KJ-2013-009).

\section{References}

1. Zhang J, Li Y, Chen X, et al: Autophagy is involved in anticancer effects of matrine on SGC-7901 human gastric cancer cells. Oncol Rep 26: 115-124, 2011.

2. Dai ZJ, Gao J, Ji ZZ, et al: Matrine induces apoptosis in gastric carcinoma cells via alteration of Fas/FasL and activation of caspase-3. J Ethnopharmacol 123: 91-96, 2009.

3. Dai ZJ, Gao J, Wu WY, et al: [Effect of matrine injections on invasion and metastasis of gastric carcinoma SGC-7901 cells in vitro]. Zhong Yao Cai 30: 815-819, 2007.

4. Luo C, Zhong HJ, Zhu LM, et al: Inhibition of matrine against gastric cancer cell line MNK45 growth and its anti-tumor mechanism. Mol Biol Rep 39: 5459-5464, 2012.

5. Luo C, Zhu Y, Jiang T, et al: Matrine induced gastric cancer MKN45 cells apoptosis via increasing pro-apoptotic molecules of Bcl-2 family. Toxicology 229: 245-252, 2007.

6. Liu YQ, Li Y, Qin J, et al: Matrine reduces proliferation of human lung cancer cells by inducing apoptosis and changing miRNA expression profiles. Asian Pac J Cancer Prev 15: 2169-2177, 2014. 
7. Niu H, Zhang Y, Wu B, Zhang Y, Jiang H and He P: Matrine induces the apoptosis of lung cancer cells through downregulation of inhibitor of apoptosis proteins and the Akt signaling pathway. Oncol Rep 2014.

8. Jiang H, Hou C, Zhang S, et al: Matrine upregulates the cell cycle protein E2F-1 and triggers apoptosis via the mitochondrial pathway in K562 cells. Eur J Pharmacol 559: 98-108, 2007.

9. Zhang S, Zhang Y, Zhuang Y, et al: Matrine induces apoptosis in human acute myeloid leukemia cells via the mitochondrial pathway and Akt inactivation. PLoS One 7: e46853, 2012.

10. Li LQ, Li XL, Wang L, et al: Matrine inhibits breast cancer growth via miR-21/PTEN/Akt pathway in MCF-7 cells. Cell Physiol Biochem 30: 631-641, 2012.

11. Zhang P, Wang Z, Chong T and Ji Z: Matrine inhibits proliferation and induces apoptosis of the androgenindependent prostate cancer cell line PC-3. Mol Med Rep 5: 783-787, 2012.

12. Zhang JQ, Li YM, Liu T, et al: Antitumor effect of matrine in human hepatoma G2 cells by inducing apoptosis and autophagy. World J Gastroenterol 16: 4281-4290, 2010.

13. Liu T, Song Y, Chen H, Pan S and Sun X: Matrine inhibits proliferation and induces apoptosis of pancreatic cancer cells in vitro and in vivo. Biol Pharm Bull 33: 1740-1745, 2010.

14. Chang C, Liu SP, Fang CH, et al: Effects of matrine on the proliferation of HT29 human colon cancer cells and its antitumor mechanism. Oncol Lett 6: 699-704, 2013.

15. Zhang Z, Wang X, Wu W, et al: Effects of matrine on proliferation and apoptosis in gallbladder carcinoma cells (GBC-SD) Phytother Res 26: 932-937, 2012.

16. Yang Z, Mu J, Chen J, et al: [Apoptosis of U937 cell line promoted by matrine through MAPK signal transduction pathway]. Zhongguo Zhong Yao Za Zhi 34: 1553-1556, 2009.

17. Zhang JW, Su K, Shi WT, et al: Matrine inhibits the adhesion and migration of BCG823 gastric cancer cells by affecting the structure and function of the vasodilator-stimulated phosphoprotein (VASP). Acta Pharmacol Sin 34: 1084-1092, 2013.

18. Ribeiro-dos-Santos A, Khayat AS, Silva A, et al: Ultra-deep sequencing reveals the microRNA expression pattern of the human stomach. PLoS One 5: e13205, 2010.

19. Chen W, Tang Z, Sun Y, et al: miRNA expression profile in primary gastric cancers and paired lymph node metastases indicates that miR-10a plays a role in metastasis from primary gastric cancer to lymph nodes. Exp Ther Med 3: 351-356, 2012.

20. Tsukamoto Y, Nakada C, Noguchi T, et al: MicroRNA-375 is downregulated in gastric carcinomas and regulates cell survival by targeting PDK1 and 14-3-3zeta. Cancer Res 70: 2339-2349, 2010.

21. Guo J, Miao Y, Xiao B, et al: Differential expression of microRNA species in human gastric cancer versus non-tumorous tissues. J Gastroenterol Hepatol 24: 652-657, 2009.

22. Yao Y, Suo AL, Li ZF, et al: MicroRNA profiling of human gastric cancer. Mol Med Rep 2: 963-970, 2009.

23. Qin S, Ai F, Ji WF, Rao W, Zhang HC and Yao WJ: miR-19a promotes cell growth and tumorigenesis through targeting SOCS1 in gastric cancer. Asian Pac J Cancer Prev 14: 835-840, 2013.

24. Ueda T, Volinia S, Okumura H, et al: Relation between microRNA expression and progression and prognosis of gastric cancer: a microRNA expression analysis. Lancet Oncol 11: 136-146, 2010.

25. Katada T, Ishiguro H, Kuwabara $Y$, et al: microRNA expression profile in undifferentiated gastric cancer. Int J Oncol 34: 537-542, 2009.

26. Li X, Luo F, Li Q, et al: Identification of new aberrantly expressed miRNAs in intestinal-type gastric cancer and its clinical significance. Oncol Rep 26: 1431-1439, 2011.

27. Wang HJ, Ruan HJ, He XJ, et al: MicroRNA-101 is downregulated in gastric cancer and involved in cell migration and invasion. Eur J Cancer 46: 2295-2303, 2010.

28. Chan SH, Wu CW, Li AF, Chi CW and Lin WC: miR-21 microRNA expression in human gastric carcinomas and its clinical association. Anticancer Res 28: 907-911, 2008.

29. Chen Z, Saad R, Jia P, et al: Gastric adenocarcinoma has a unique microRNA signature not present in esophageal adenocarcinoma. Cancer 119: 1985-1993, 2013

30. Kim BH, Hong SW, Kim A, Choi SH and Yoon SO: Prognostic implications for high expression of oncogenic microRNAs in advanced gastric carcinoma. J Surg Oncol 107: 505-510, 2013.
31. Inoue $\mathrm{T}$, Iinuma $\mathrm{H}$, Ogawa $\mathrm{E}$, Inaba $\mathrm{T}$ and Fukushima R: Clinicopathological and prognostic significance of microRNA-107 and its relationship to DICER1 mRNA expression in gastric cancer. Oncol Rep 27: 1759-1764, 2012.

32. Liu T, Tang H, Lang Y, Liu M and Li X: MicroRNA-27a functions as an oncogene in gastric adenocarcinoma by targeting prohibitin. Cancer Lett 273: 233-242, 2009.

33. Zhao X, Yang L and Hu J: Down-regulation of miR-27a might inhibit proliferation and drug resistance of gastric cancer cells. J Exp Clin Cancer Res 30: 55, 2011.

34. Matsuo M, Nakada C, Tsukamoto Y, et al: MiR-29c is downregulated in gastric carcinomas and regulates cell proliferation by targeting RCC2. Mol Cancer 12: 15, 2013.

35. Saito Y, Suzuki H, Imaeda H, et al: The tumor suppressor microRNA-29c is downregulated and restored by celecoxib in human gastric cancer cells. Int J Cancer 132: 1751-1760, 2013.

36. Osawa S, Shimada Y, Sekine S, et al: MicroRNA profiling of gastric cancer patients from formalin-fixed paraffin-embedded samples. Oncol Lett 2: 613-619, 2011.

37. Song YX, Yue ZY, Wang ZN, et al: MicroRNA-148b is frequently down-regulated in gastric cancer and acts as a tumor suppressor by inhibiting cell proliferation. Mol Cancer 10: 1, 2011.

38. Chen G, Shen ZL, Wang L, Lv CY, Huang XE and Zhou RP: Hsa-miR-181a-5p expression and effects on cell proliferation in gastric cancer. Asian Pac J Cancer Prev 14: 3871-3875, 2013.

39. Zhang X, Nie Y, Du Y, Cao J, Shen B and Li Y: MicroRNA-181a promotes gastric cancer by negatively regulating tumor suppressor KLF6. Tumour Biol 33: 1589-1597, 2012.

40. Carvalho J, van Grieken NC, Pereira PM, et al: Lack of microRNA-101 causes E-cadherin functional deregulation through EZH2 up-regulation in intestinal gastric cancer. J Pathol 228: 31-44, 2012.

41. Zhu YM, Zhong ZX and Liu ZM: Relationship between let-7a and gastric mucosa cancerization and its significance. World $\mathrm{J}$ Gastroenterol 16: 3325-3329, 2010.

42. Yang Q, Jie Z, Cao H, et al: Low-level expression of let-7a in gastric cancer and its involvement in tumorigenesis by targeting RAB40C. Carcinogenesis 32: 713-722, 2011.

43. Zhu Y, Xiao X, Dong L and Liu Z: Investigation and identification of let-7a related functional proteins in gastric carcinoma by proteomics. Anal Cell Pathol (Amst) 35: 285-295, 2012.

44. Chiang Y, Zhou X, Wang Z, et al: Expression levels of microRNA-192 and -215 in gastric carcinoma. Pathol Oncol Res 18: 585-591, 2012.

45. Song Y, Zhao F, Wang Z, et al: Inverse association between miR-194 expression and tumor invasion in gastric cancer. Ann Surg Oncol 19 Suppl 3: S509-517, 2012.

46. Liu ZS, Luo ZQ, Xie B, et al: [Effect and mechanism of matrine on apoptosis of Raji cells]. Zhong Yao Cai 34: 576-579, 2011.

47. Lu J, Luo Q, Cheng P, Liu X, Bai M and Tu M: [The Role of Matrine and Mitogen-Ativated Protein Kinase/Extracellular Signal-Regulated Kinase Signal Transduction in the Inhibition of the Proliferation and Migration of Human Umbilical Veins Endothelial Cells Induced by Lung Cancer cells.]. Zhongguo Fei Ai Za Zhi 12: 747-752, 2009.

48. Guo L, Xue TY, Xu W and Gao JZ: Matrine promotes G0/G1 arrest and down-regulates cyclin D1 expression in human rhabdomyosarcoma cells. Panminerva Med 55: 291-296, 2013.

49. Chen Q, Liu L and Cao H: [Effects of matrine on the growth inhibition, c-myc and hTERT protein expression in human adenocarcinoma lung cancer cell line A549.]. Zhongguo Fei Ai Za Zhi 11: 559-562, 2008.

50. Huang FX, Zhang Y and Wang WJ: [Effects of matrine on cgi-100 gene expression and proliferation in K562 cells]. Zhongguo Shi Yan Xue Ye Xue Za Zhi 16: 525-530, 2008.

51. Bae S, Lee EM, Cha HJ, et al: Resveratrol alters microRNA expression profiles in A549 human non-small cell lung cancer cells. Mol Cells 32: 243-249, 2011.

52. Sethi S, Li Y and Sarkar FH: Regulating miRNA by natural agents as a new strategy for cancer treatment. Curr Drug Targets 14: 1167-1174, 2013 